\title{
DESIGN OF AN ARTIFICIAL NEURAL NETWORK FOR THE ANALYSIS OF STELLAR SPECTRA
}

\author{
JAIME KLAPP ${ }^{1}$, CELIA R. FIERRO-SANTILLAN ${ }^{1}$, LEONARDO DI G SIGALOTTI ${ }^{2}$ \\ AND ISIDORO GITLER ${ }^{3}$ \\ ${ }^{1}$ Departamento de Física, Instituto Nacional de Investigaciones Nucleares (ININ) \\ Carretera México-Toluca km. 36.5, La Marquesa, 52750 Ocoyoacac, Estado de México, Mexico \\ jaime.klapp@inin.gob.mx, celia.fierro.estrellas@gmail.com \\ 2 Área de Física de Procesos Irreversibles, Departamento de Ciencias Básicas, Universidad Autónoma \\ Metropolitana-Azcapotzalco (UAM-A), Av. San Pablo 180, 02200 México City, México \\ leonardo.sigalotti@gmail.com \\ ${ }^{3}$ Departamento de Matemáticas, Centro de Investigación y de Estudios Avanzados CINVESTAV-IPN \\ Apartado Postal 14-740 07000 México City, D.F. \\ igitler@math.cinvestav.edu.mx
}

Key words: Artificial neural network, stellar atmospheres, techniques: spectroscopy

\begin{abstract}
We have developed an artificial neural network, whose purpose is to automatically find in a database of synthetic stellar spectra the one which best reproduces an observed spectrum. Using the equivalent widths of selected spectral lines, the network fits a set of lines related to the physical parameters in the stellar atmosphere (i.e., temperature, gravity and mass loss rate). The main advantage of this approach is its scalability.
\end{abstract}

\section{INTRODUCTION}

All our information about the physical conditions and the chemical composition of stars comes from the study of their electromagnectic spectrum. In massive stars $\left(20 M_{\odot} \leq M_{\star} \leq 120 M_{\odot}\right)$, we can identify three components of stellar spectrum: the continuum, the absorption and the emission lines. They can give us information about the structure of the atmosphere, since they come from layers located at different depths in the stellar atmosphere (e.g., see Figure 1).

The spectrum emitted by the stellar interior where the atmosphere begins is called the continuum. Due to its strong dependence on temperature and the energy distribution, the continuum can be modeled by Planck's law of blackbody radiation ${ }^{1}$.

The absorption lines come from the base of the atmosphere. This region is in local thermodynamic equilibrium (LTE). The atoms in the atmosphere absorb certain characteristic wavelengths of the continuum

\footnotetext{
${ }^{1} \mathrm{~A}$ blackbody is an ideal radiator that does not exist in the real world. However, many objects, including stars, behave as a blackbody. Blackbody radiation can be produced in a closed cavity whose walls absorb all radiation incident upon them and coming from inside the cavity. The walls and the radiation in the cavity are in equilibrium. Both are at the same temperature, and the walls emit all the energy they receive.
} 

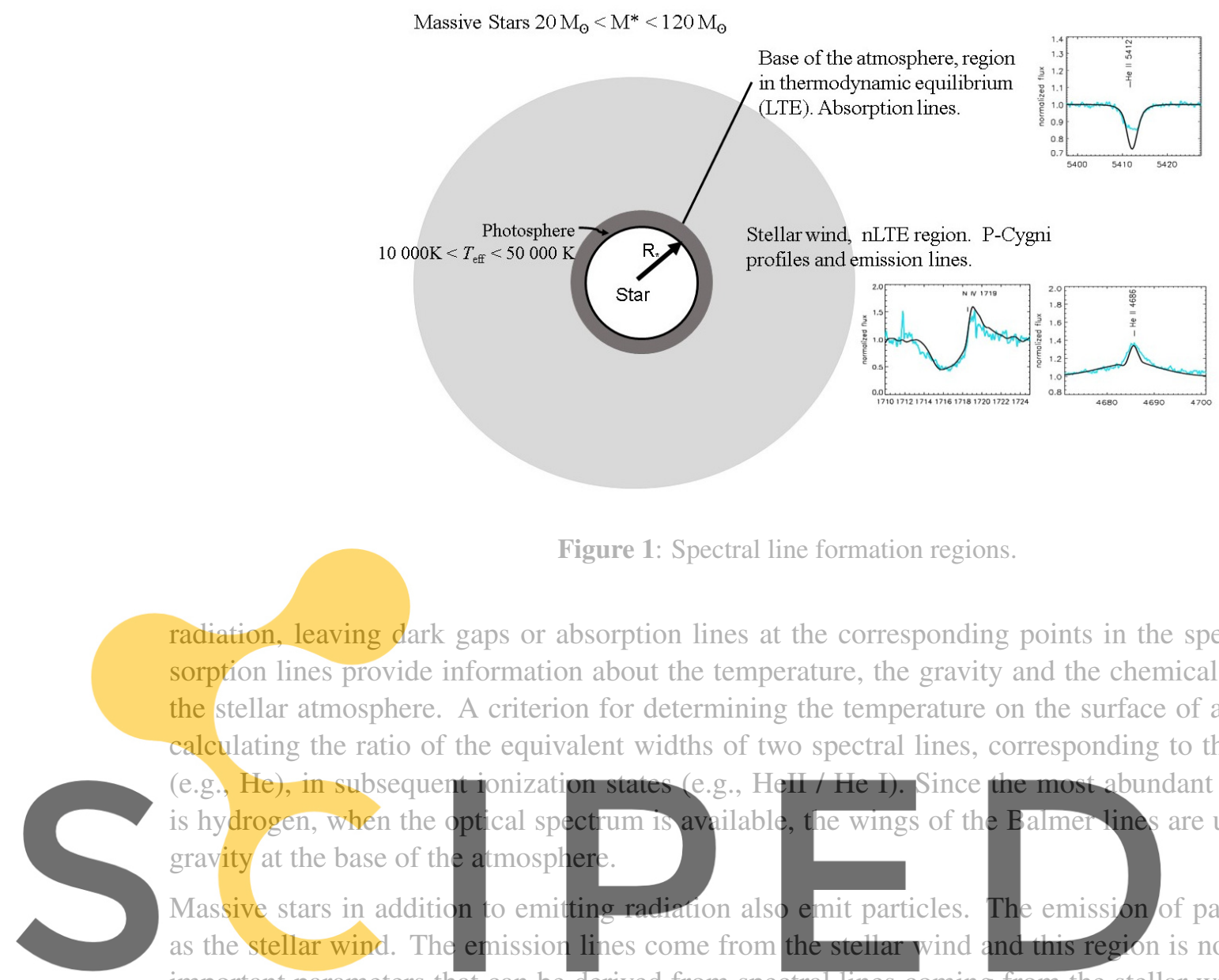

Figure 1: Spectral line formation regions.

radiation, leaving dark gaps or absorption lines at the corresponding points in the spectrum. The absorption lines provide information about the temperature, the gravity and the chemical composition on the stellar atmosphere. A criterion for determining the temperature on the surface of a star consists of calculating the ratio of the equivalent widths of two spectral lines, corresponding to the same element is hydrogen, when the opto gravity at the base of the a

Massive stars in addition as the stellar wind. The
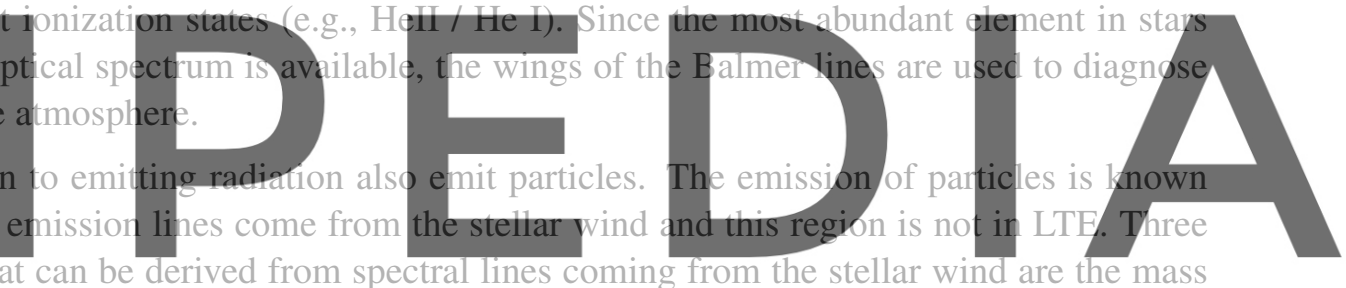
important parameters that can be derived from spectral lines coming from the stellar wind are the mass

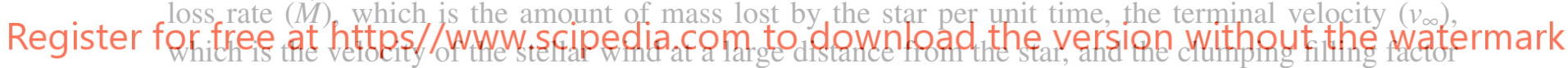

$\left(f_{c l}\right)$, which is the average fraction of the volume in regions filled with material, called the clumps, by assuming that the volume between clumps is empty. Stars with mass loss rates on the order of $10^{-6} M_{\odot}$ $\mathrm{yr}^{-1}$, or higher, show emission lines in their optical spectrum. The best known are the $H_{\alpha}$ and $H_{\beta}$ lines in O- and B-type stars. The P-Cygni profiles in the UV spectrum have been studied quantitatively by various authors to derive information on the $\dot{M}, v_{\infty}$ and $f_{c l}$.

A model of stellar atmosphere is a specification of the state of all the parameters that govern the physical phenomena that occur there. Since the problem is very complex, we cannot construct analytic solutions. Therefore, we discretize a coordinate system and solve the equations in several tens to hundred of points distributed in the atmosphere. The number of parameters depend on the type of the model. In recent years a significant progress has been achieved in the field of stellar atmospheres. A self-consistent analysis of spectral regions from the X-ray to the IR is now possible, thanks to the fertile combination of the large amount of observational data and the availability of sophisticated stellar atmosphere codes. Space- and Earth-based telescopes are generating large amounts of astronomical data. The Far Ultraviolet 

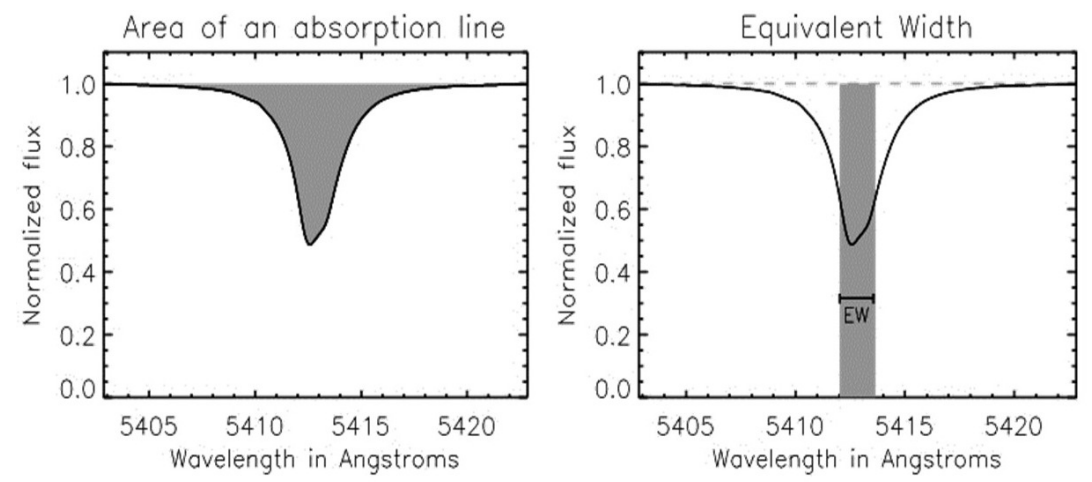

Figure 2: Equivalent width of a spectral line.

Spectroscopic Explorer (FUSE) ${ }^{2}$, the International Ultraviolet Explorer (IUE) ${ }^{3}$ in ultraviolet (UV), the Hubble Space Telescope (HST) ${ }^{4}$ in UV and optical, and the Sloan Digital Sky Survey (SDSS) ${ }^{5}$ [1] in optical e IR, to mention a few, have generated databases of stellar spectra on the order of the petabytes. On the other hand, there are databases of synthetic stellar spectra with tens, hundreds and even thousands of stellar models, with their corresponding synthetic spectra in the optical, UV and IR (see, for example, the grids for Wolf-Rayet (WR) stars [2], the POLLUX database [3] and the grids of models for OB stars $[4,5,6]$ calculated with the CMFGEN code [7]. Such databases of theoretical spectra have produced data volumes on the order of the terabytes. This data volume has contributed to bring the era of Big Data to the field of spectroscopic analysis, requining the creation of new computational tools capable of the management of large volumes of data, maintaining the quality of the analysis.

2 MEASUREMENT OF SPECTRAL LINES

The most basic thing we can measure for a spect
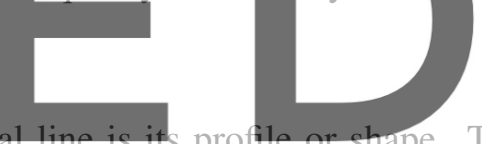

$(\mathrm{EW})$ is a measure of the total absortion or emission in a line. It is defined as the width of a rectangle

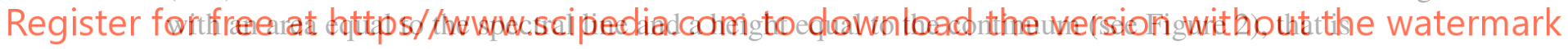

$$
E W=\frac{\int F_{0}-F_{\mathrm{v}} d v}{F_{0}}
$$

In order to measure the EWs, we developed the Spectral Line Analysis Module (SLAM) [8]. This program works with normalized synthetic and observed spectra, provided in a comma-separated values (CSV) files, generates CSV files with the parameters of a spectral line, measured in hundreds or thousands of spectra, discriminating between absorption or emission lines, and calculates its EW.

\footnotetext{
${ }^{2}$ The Far Ultraviolet Spectroscopic Explorer (FUSE) was a space-based telescope operated by The Johns Hopkins University to detect light in the far-ultraviolet portion of the spectrum.

${ }^{3}$ IUE was an international collaboration of NASA, the European Space Agency (ESA), and the United Kingdom's Science and Engineering Research Council (SERC; now Particle Physics and Astronomy Research Council, or PPARC ).

${ }^{4} \mathrm{HST}$ is a program of the European Space Agency (ESA), the National Aeronautics, and Space Administration (NASA)

${ }^{5}$ The SDSS used a dedicated 2.5-meter telescope at Apache Point Observatory, New Mexico. SDSS is supported by the Alfred P. Sloan Foundation, the Participating Institutions, and the U.S. Department of Energy Office of Science.
} 


\section{The FITspec Code under a neural network architecture.}

The FITspec code is a software that automatically finds in a database the synthetic spectrum that best reproduces the equivalent widths (EWs) measured in an observed spectrum (Fierro-Santillán et al. 2018). The program was developed in Python, under a functional programming approach. FITspec is based on the deviations or errors of the EWs of the spectral lines in the models, with respect to the observed spectra. This problem is suitable to be solved under an artificial neural network (ANN) approach. We have chosen to use an ANN with three layers (input, hidden and output) architecture as shown in Fig. 3 ), with unsupervised learning due to the impossibility for an astronomer to compare by eye an observed spectrum with thousands of models. Therefore, it is not possible to supply training data to the ANN. The ANN finds among the thousands of models in a database the one whose spectral lines have the greatest similarity to an observed spectrum, based on their EWs.

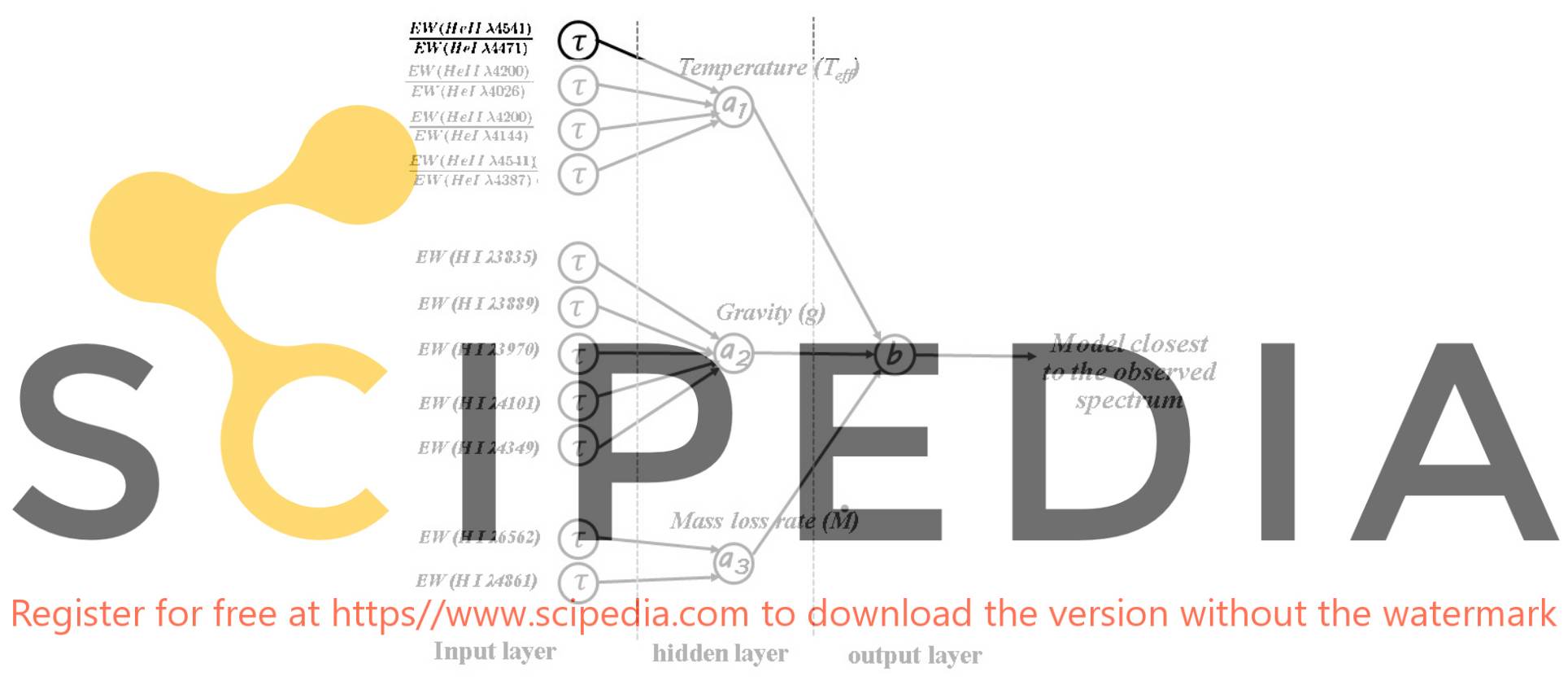

Figure 3: Structure of the artificial neural network.

Input layer. Each node in this layer calculates how far the values of a model are with respect to the values of the observed spectrum (desired values). The input values can be EWs or ratios of two EWs. In the case of EWs, the tolerance $(\tau)$ is calculated as

$$
\tau_{\mathrm{EW}}=\left|\frac{\mathrm{EW}_{\mathrm{obs}}-\mathrm{EW}_{\text {automatic }}}{\mathrm{EW}_{\mathrm{obs}}}\right|,
$$

while in the case of ratios

$$
\tau_{\text {ratio }}\left(\frac{E W_{1}}{E W_{2}}\right)=\left|\frac{\left(\frac{E W_{1}}{E W_{2}}\right)_{\mathrm{obs}}-\left(\frac{E W_{1}}{E W_{2}}\right)_{\text {model }}}{\left(\frac{E W_{1}}{E W_{2}}\right)_{\mathrm{obs}}}\right| .
$$


Hidden layer. The tolerance values are collected from the input layer and put into a sigmoid function

$$
a_{i}=\frac{1}{1+\exp \sum_{j=1}^{n} \theta_{i j}^{1} \tau_{i j}},
$$

where $\theta_{i j}$ are the weights on the tolerances $\tau_{j}$. Each node in the hidden layer uses a set of tolerances corresponding to a set of spectral lines, which are traditionally used by astronomers as a criterion to calculate the stellar parameters, namely $T_{\text {eff }}, \log g$ and $\dot{M}$. Table 1 lists the parameters, the criteria and the lines that were taken into account in the design of the ANN.

Table 1: Criteria for the activation function of the ANN

\begin{tabular}{|c|c|c|}
\hline Parameter & Criterion & Suitable lines \\
\hline$T_{\text {eff }}$ & Ratios of line & He II $\lambda 4541$ / He I $\lambda 4471$ \\
\hline
\end{tabular}

He II $\lambda 4200 /$ He I $\lambda 4144$ He II $\lambda 4541 /$ He I $\lambda 4387$

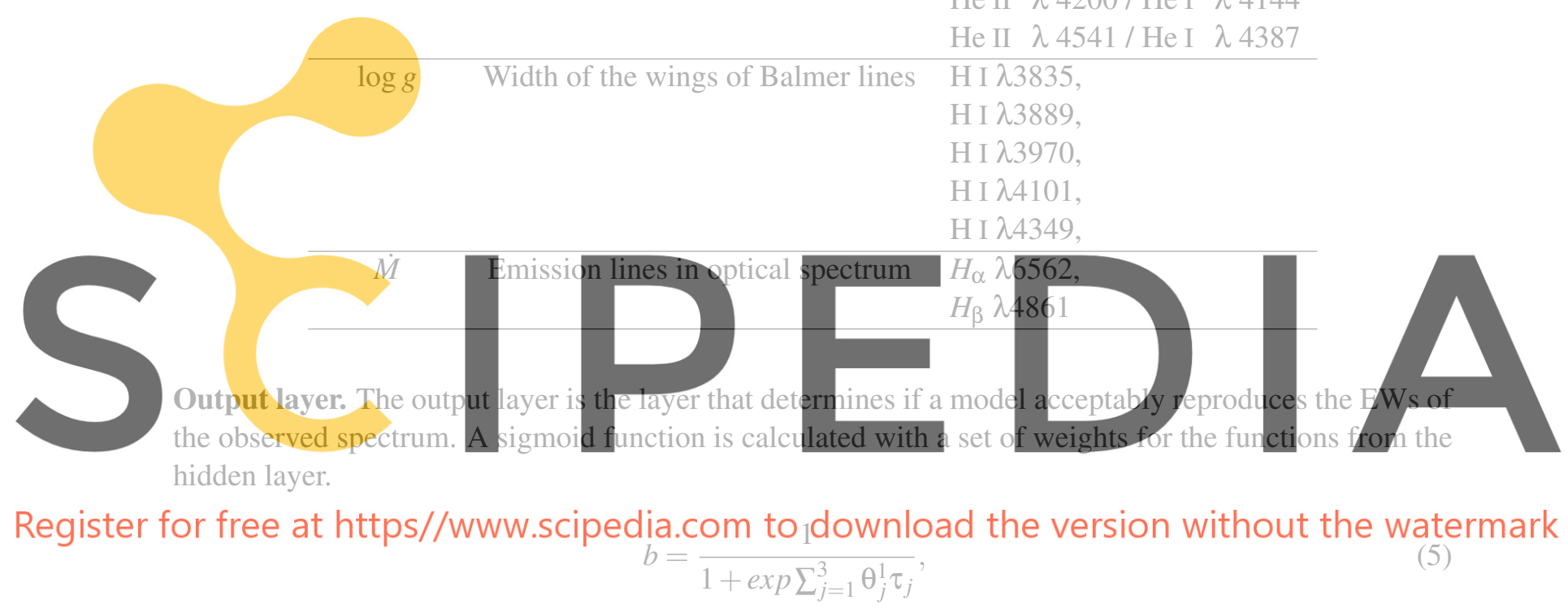

A detailed analysis of a stellar atmosphere involves physical parameters, both at the base of the atmosphere and in the wind, where each parameter is based on a set of spectral lines. Additionally, the chemical composition of the star requires the study of several lines of the same element. The number of lines to analyze can make the analysis unmanageable. The ANN design allows the coupling of new sets of lines, adding neurons in the input and hidden layers, which will allow an increasingly complete and detailed analysis of the observed spectra. The EW-based approach allows the ANN to be independent of the stellar atmosphere code or database used to create the synthetic spectra.

\section{Summary}

Spectroscopy applied to the study of stellar spectra has had a great boost thanks to the use of supercomputers, which allow the calculation of hundreds or thousands of models in parallel. The large volume of observational and theoretical data make it necessary to develop software tools capable of finding solutions using large databases. We have previously created FITspec, a program capable of exploring a 
database with thousands of models and finding the one that best reproduces an observed spectrum, using funcional programming. In order to solve this problem an artificial neural network has been designed. One main advantage of this approach is its scalability.

\section{Acknowledgements}

This work has been partially supported by the European Union Horizon 2020 programme under the ENERXICO Project, Grant Agreement No. 828947 and the Mexican CONACYT-SENER-Hidrocarburos Grant Agreement No. B-S-69926. The calculations of this paper were performed using the supercomputing facilities of ABACUS-Laboratorio de Matemática Aplicada y Cómputo de Alto Rendimiento of Cinvestav-IPN and the Barcelona Supercomputing Center (BSC).

\section{REFERENCES}

[1] Gunn, J. E., Siegmund, W. A., Mannery, E. J., et al. 2006, AJ, 131, 2332

[2] Hamann, W.-R., \& Gräfener, G. 2004, A\&A, 427, 697

[3] Palacios, A., Gebran, M., Josselin, E., Martins, F., Plez, B., Belmas, M., \& Lèbre, A. 2010, A\&A, 516, A13

[4] Fierro, C. R., Borissova, J., Zsargó, J., Díaz-Azuara, A., Kurtev, R., Georgiev, L., Ramírez-Alegría, S., \& Peñaloza, F., 2015, PASP, 127, 428

[5] Zsargó, J., Arrieta, A., Fierro, C., Klapp, J., Hillier, D. J., Arias, L., Mendoza, J., \& Georgiev, L. N.

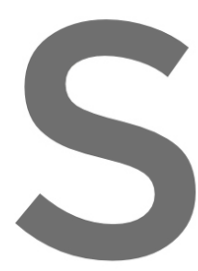

2017, in Astronomienl Society of the Pacific S. Zharikov, D. Korčáková, \& M. Wolf, 407

[6] Zsargó, J., Fierro-Santillán, C., Klapp, J., Arr Hareter, M., and Puebla, R.

[7] HiHlier, D. J. \& Miller, D. L. 1998, ApJ, 496,
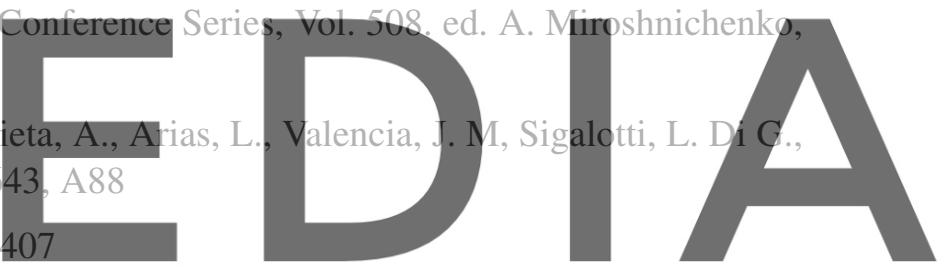

[8] Fierro, C., Klapp, J., Zsargó, J., Sigalotti, L. Di G., Hareter, M., 2021, The Astronomical Journal, Register for freẹasshttps//www.scipedia.com to download the version without the watermark 